\title{
Activation of the NLRC4 inflammasome in renal tubular epithelial cell injury in diabetic nephropathy
}

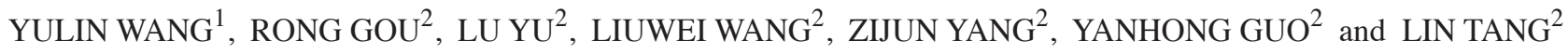 \\ ${ }^{1}$ Department of Nephrology, Zhengzhou University, Zhengzhou, Henan 450000; ${ }^{2}$ Department of Nephrology, \\ The First Affiliated Hospital of Zhengzhou University, Zhengzhou, Henan 450052, P.R. China
}

Received December 22, 2020; Accepted May 4, 2021

DOI: $10.3892 /$ etm.2021.10246

\begin{abstract}
Renal tubular interstitial injury plays a key role in the progression of diabetic nephropathy (DN) and, thus, the study of renal tubular injury in DN is important. The aim of the present study was to elucidate the role of the NLR family CARD domain containing 4 (NLRC4) inflammasome in renal tubular epithelial cell (RTEC) injury in DN. Human kidney biopsy tissues were obtained from patients with DN, and normal kidney tissues were obtained from nephrectomies performed for renal hamartoma. Human RTECs (HK2 cells) were divided into normal glucose (D-glucose $5.6 \mathrm{mmol} / \mathrm{l}$ ), high glucose (HG; $30 \mathrm{mmol} / \mathrm{l}$ ), high osmotic (D-glucose $5.6 \mathrm{mmol} / \mathrm{l}+\mathrm{D}$-mannitol $24.4 \mathrm{mmol} / \mathrm{l}), \mathrm{HG}+\mathrm{NLRC} 4$ small interfering (si)RNA or HG + siRNA control groups. Then, the expression levels of NLRC4, PTEN-induced kinase 1 (PINK1) and parkin, as well as the levels of mitochondrial reactive oxygen species, which are associated with mitophagy, were observed. The expression levels of NLRC4, PINK1, parkin and phosphorylated parkin in the RTECs of patients with DN were higher compared with those in normal controls. In HK2 cells, HG stimulated the expression of NLRC4, the secretion of IL-1 $\beta$ and IL-18 and cell death. Moreover, knockdown of NLRC4 expression in HK2 cells treated with HG reduced the secretion of the inflammatory cytokines, IL-1 $\beta$ and IL-18. The findings of the present study may provide a rationale for the development of treatments for patients with DN by preventing inflammasome activation.
\end{abstract}

\section{Introduction}

With the development of the economy and changes in lifestyle, the incidence of diabetes, particularly type 2 diabetes, is increasing in China, and has become a major public health

Correspondence to: Dr Lin Tang, Department of Nephrology, The First Affiliated Hospital of Zhengzhou University, 1 Jianshe Road, Zhengzhou, Henan 450052, P.R. China

E-mail: wangyulin1216@163.com

Key words: NLRC4 inflammasome, mitophagy, renal tubular epithelial cells, diabetic nephropathy problem threatening the health of the Chinese population (1). The latest data released by the International Diabetes Federation in 2019 revealed that the number of patients with diabetes worldwide had reached 463 million, and the number of patients with diabetes in China was 116.4 million, accounting for $\sim 1 / 4$ of the total number of patients worldwide (2). Diabetic nephropathy (DN) is not only a serious complication of diabetes, but is also one of the most important causes of end-stage renal disease (ESRD) (3). Some investigators have reported that DN has become the leading cause of chronic kidney disease among hospitalized patients in China (4). At present, there is a large number of studies and recommended treatment approaches to diabetic nephropathy (DN); however, studies in the United States over the past 20 years indicate that the incidence of ESRD caused by diabetes has not significantly decreased (5). Thus, effective intervention measures to delay the progression of DN are currently lacking. Therefore, studying the pathogenesis of DN is crucial for further identifying effective treatments.

The pathological changes of DN include glomerulosclerosis, tubulointerstitial fibrosis and renal vascular lesions. At present, studies on the mechanism of DN are mostly focused on glomerular injury, and the clinical indices used to assess the severity of DN are based on the changes of glomerular structure and function (6). However, the renal tubulointerstitium accounts for $>90 \%$ of the renal parenchyma and serves a variety of important functions (7). Renal tubular interstitial injury plays a key role in the progression of DN and, therefore, its study is important.

In recent years, it has been reported that inflammasomes serve an important role in the inflammatory response and cellular injury of DN (8-10). Inflammasomes are a class of large polyprotein complexes, the receptor proteins of which include Nod-like receptors (NLR) family pyrin domain containing (NLRP)1, NLRP3 and NLR family CARD domain containing 4 (NLRC4) from the NLR family, and absent in melanoma 2 from the HIN-200 family, which can activate caspase-1, regulate IL-1 $\beta$ and IL-18 maturation and secretion, and trigger inflammation and cell injury (11). Some bacterial infections can activate NLRC4 inflammatory bodies, causing an inflammatory response (12). Moderate inflammation is beneficial for the clearance of bacteria and can inhibit bacterial infection, while excessive inflammation may lead to host cell death. Previous studies reported that NLRC4 
inflammasomes are also involved in the pathological process of kidney disease. For example, Yuan et al (13) observed a significant increase in NLRC4 expression in the renal tubules and interstitium of patients with DN compared with that of control cases. Quantitatively, NLRC4 staining intensity exhibited a one-fold increase in the renal tubulointerstitium of patients with DN (13). In addition, it has been revealed that the expression of NLRC4 in intestinal epithelial cells is increased and it is involved in intestinal inflammation $(14,15)$. The main aim of the present study was to examine the role of the NLRC4 inflammasome in renal tubular epithelial cell (RTEC) injury in DN.

Mitophagy is a type of selective autophagy, which was first proposed by Lemasters in 2005 (16). Mitophagy selectively removes damaged or unwanted mitochondria via PTEN-induced kinase 1 (Pink1)/parkin and NIP-3-like protein $\mathrm{X} / \mathrm{BCL} 2$ interacting protein 3 signaling (17). This process involves autophagosome fusion with lysosomes, degradation of damaged or dysfunctional mitochondria and maintenance of reactive oxygen species (ROS) balance, and it participates in a variety of pathophysiological processes and diseases (18).

Mitochondrial ROS (mROS) produced by mitophagy can activate the NLRP3 inflammasome (19). As a member of the NLR family, NLRC4 has a similar structure to NLRP3 and may also be activated by mROS. Recent studies have reported that mitophagy can activate the NLRC4 inflammasome, causing an inflammatory response and cell death in macrophages infected by Pseudomonas aeruginosa and in myocardial cells following myocardial infarction in a high-glucose (HG) environment $(20,21)$. Therefore, the NLRC4 inflammasome may be an important factor mediating mitophagy in $\mathrm{DN}$; however, to the best of our knowledge, there is no related research at present. The present study was undertaken to investigate whether mitophagy dysfunction and NLRC4 inflammasome activation in RTECs are involved in the inflammatory response and apoptosis in a HG environment.

\section{Materials and methods}

Morphological analysis of human kidney samples. Human kidney biopsy tissues of patients $(n=42)$ with type $2 \mathrm{DN}$ were obtained from the Department of Nephrology of The First Affiliated Hospital of Zhengzhou University (Zhengzhou, China), and normal kidney tissues from nephrectomies performed for renal hamartoma $(n=10)$ served as the control (the kidney samples in paraffin blocks were obtained retrospectively between 01/2016-10/2018 and the experiments were performed in 11/2018). All renal tissues were cut into sections $\sim 2 \mu \mathrm{m}$ thick and examined via immunofluorescence, light microscopy (LM) and electron microscopy (EM), according to standard methods. The sections were evaluated according to the pathological classification standard of DN published in the Journal of American Society of Nephrology in 2010 (22) as follows: Class I: Mild or non-specific LM changes and EM-proven glomerular basement membrane (GBM) thickening, biopsy does not meet any of the criteria mentioned below for class II, III or IV, GBM>395 nm in female and $>430 \mathrm{~nm}$ in male individuals aged $\geq 9$ years; class IIa: Mild mesangial expansion in $>25 \%$ of the observed mesangium; class IIb: Severe mesangial expansion in $>25 \%$ of the observed mesangium; class III: At least one convincing Kimmelstiel-Wilson lesion, does not meet criteria for class IV; and class IV: Global glomerular sclerosis in $>50 \%$ of glomeruli. The present study has been approved by the Ethics Committee of The First Affiliated Hospital of Zhengzhou University (Zhengzhou, China; approval no. 2018-KY-023).

Blood and urine examination. The blood and urine samples of the patients were collected for the detection of $24 \mathrm{~h}$ urinary protein quantity, hemoglobin, glycosylated hemoglobin (HbA1c), serum creatinine, serum albumin and estimated glomerular filtration rate (eGFR). The eGFR was calculated using the chronic kidney disease epidemiology collaboration formula (23) based on serum creatinine.

Cell culture. Human RTECs (HK2 cells) were obtained from The Cell Bank of Type Culture Collection of The Chinese Academy of Sciences, and cultured in DMEM (Invitrogen; Thermo Fisher Scientific, Inc.) supplemented with $5.6 \mathrm{mM}$ D-glucose, 10\% FBS (Invitrogen; Thermo Fisher Scientific, Inc.), $100 \mathrm{U} / \mathrm{ml}$ penicillin and $100 \mu \mathrm{g} / \mathrm{ml}$ streptomycin in a $5 \% \mathrm{CO}_{2}$ incubator at $37^{\circ} \mathrm{C}$.

To detect the effect of HG on NLRC4 inflammasome and mitophagy, cells were cultured under normal glucose (NG) conditions (5.6 mM glucose), hyperosmotic (HO) conditions (5.6 mM glucose $+24.4 \mathrm{mM}$ mannitol) or $\mathrm{HG}$ conditions (30 $\mathrm{mM}$ glucose) for $48 \mathrm{~h}$.

Small interfering (si)RNA transfection. HK2 cells were transfected with scramble small interfering RNA (siRNA) and NLRC4 siRNA (Sangon Biotech Co., Ltd.) using Lipofectamine $^{\circledR} 2000$ (Thermo Fisher Scientific, Inc.) according to the instructions of the manufacturer. In total, $0.1 \mathrm{nmol}$ siRNA and $5 \mu 1$ Lipofectamine ${ }^{\circledR} 2000$ were diluted in $250 \mu$ l Opti-MEM (Invitrogen; Thermo Fisher Scientific, Inc.), and incubated for $5 \mathrm{~min}$ at room temperature. The sequence of the siRNAs was as follows: NLRC4 siRNA forward, 5'-CCU UAUUACCUCAUCGAAUTT-3' and reverse, 5'-AUUCGA UGAGGUAAUAAGGTT-3'; control siRNA forward, 5'-UUC UCCGAACGUGUCACGUTT-3' and reverse, 5'-ACGUGA CACGUUCGGAGAATT-3'. Then, the two diluents were mixed and incubated for $20 \mathrm{~min}$ at room temperature. The resulting DNA-Lipofectamine ${ }^{\circledR} 2000$ complex was added to $5 \times 10^{5}$ cells and incubated at $37^{\circ} \mathrm{C}$ for $6 \mathrm{~h}$. Then, the medium was replaced with DMEM supplemented with $2 \mathrm{mmol} / 1$ glutamine and 10\% FBS.

Western blotting. After cleavage, the renal homogenates and cells in different groups was extracted using a protein extraction kit (Qiagen China Co., Ltd.), and the total protein content was determined using the BCA method. The $10 \%$ gel for SDS-PAGE was prepared, the target protein sample was collected and adjusted to the same concentration. The lanes on both sides of the sample were sampled with $1 \mathrm{X}$ loading buffer at an equal volume, and the marker was adjusted to the same volume as the sample using $1 \mathrm{X}$ loading buffer. Electrophoresis was performed and the target protein was moved to the end at $1 \mathrm{~cm}$ above the lower edge of the gel. After electrophoresis, the proteins were transferred to a PVDF membrane, followed by blocking at room temperature for $1 \mathrm{~h}$ and incubated overnight at $4^{\circ} \mathrm{C}$ with the primary antibodies. 
The membrane was washed three times with TBS with $0.05 \%$ Tween-20 (TBST) and then incubated at room temperature for $2 \mathrm{~h}$ with the secondary antibody. The membrane was washed again with TBST to elute the primary antibody. HRP HR and the ECL method (Wuhan Boster Biological Technology, Ltd.) were used to visualize the bands. The following antibodies were used for protein detection: Anti-NLRC4 (cat. no. ab99860; 1:1,000), anti-parkin (cat. no. ab233434; 1:500), anti-phosphorylated (p)-parkin (cat. no. ab73015; 1:500), anti-PINK1 (cat. no. 23707; 1:500) and anti- $\beta$-actin (cat. no. ab8227; 1:1,000). All antibodies were purchased from Abcam. Each experiment was performed in triplicate.

Reverse transcription-quantitative (RT-q)PCR analysis. The renal homogenates and cells of different groups were cleaved and RNA was extracted using an RNA extraction kit (Qiagen China Co., Ltd.). Total RNA was determined using a micro ultraviolet spectrophotometer. Using total RNA as the template, a random primer hexamer (Shenggong Bioengineering Shanghai Co., Ltd.) was used as the reverse primer, and a total of $40 \mu \mathrm{l}$ cDNA was synthesized using PrimeScript ${ }^{\mathrm{TM}}$ II High Fidelity RT-PCR Kit (Takara Biotechnology Co, Ltd.) according to the manufacturer's protocol. Fluorescence qPCR amplification was performed as follows: The PCR amplification reaction system was $50 \mu l$, and the ratio of each detection index to the internal reference GAPDH was used to indicate the mRNA expression level of the gene. The expression levels of tested genes were normalized to that of human GAPDH and calculated using the $2^{-\Delta \Delta \mathrm{Cq}}$ method (24). The assay was performed in triplicate. The primers for the NLRC4 gene were as follows: Forward, 5'-TCTACCTGATCCAGCATTAGT CAG-3' and reverse, 5'-TGCCACCCAACAAGCCTAGC-3'. GAPDH was used as a reference gene, and the primers were as follows: Forward, 5'-TCAACAGCGACACCCACTCC-3' and reverse, 5'-TGAGGTCCACCACCCTGTTG-3' (25).

ELISA. IL-1 $\beta$ and IL-18 levels in cell culture supernatants and urine were determined using ELISA kits, according to the manufacturer's instructions (Genmed Scientifics, Inc.). Each experiment was performed in triplicate.

Measurement of superoxide generation and apoptosis. Briefly, mitochondrial superoxide generation was detected using MitoSOX Red indicator (Invitrogen; Thermo Fisher Scientific, Inc.). Mitochondrial-associated ROS levels were measured by staining cells with MitoSOX $(2.5 \mathrm{mM})$ for $30 \mathrm{~min}$ at $37^{\circ} \mathrm{C}$. Cells were then washed with PBS solution and resuspended in cold PBS solution containing $1 \%$ FBS. TUNEL assay was used to measure cell apoptosis, according to the manufacturer's instructions (cat. no. GS0246; Beijing Baiao Laibo Technology Co., Ltd.).

Statistical analysis. Statistical analyses were performed with SPSS 20.0 software (IBM Corp). Data are presented as the mean $\pm \mathrm{SD}$, unless otherwise indicated. The difference between means was assessed using the unpaired Student's t-test for two-group comparisons. Classification variables are described as percentages. Other data from experiments were analyzed using one-way ANOVA where appropriate. Both LSD and SNK post hoc tests were used for the ANOVA analysis of the PCR results of mitophagy-related indices, NLRC4, IL-1 $\beta$, IL-18 and cell apoptosis in different groups. $\mathrm{P}<0.05$ was considered to indicate a statistically significant difference.

\section{Results}

Clinical characteristics of patients with type $2 \mathrm{DN}$ and controls. The pathological types of DN were classified as class I ( $n=8)$, IIa $(n=11)$, IIb $(n=10)$, III $(n=9)$ and IV $(n=4)$. The levels of urinary protein and $\mathrm{HbA} 1 \mathrm{C}$ in the $\mathrm{DN}$ group were higher compared with those in the control group. Moreover, patients with DN class IV had the most severely compromised renal function and the lowest hemoglobin levels. With the aggravation of the pathological injury of DN, renal function deteriorated, and anemia worsened (Table I).

NLRC4 expression is increased, while the expression levels of PINK1 and parkin are decreased in the renal tissues of patients with DN. A total of 42 renal tissue samples were collected from patients with DN confirmed by renal biopsy, and 10 normal renal tissue samples obtained at a distance of $3-5 \mathrm{~cm}$ from the edge of renal hamartoma served as the control sample. Renal tubular atrophy and interstitial fibrosis were observed via LM, and immunohistochemistry was used to observe the expression and localization of NLRC4 in the renal tissues of patients with DN. The results of immunohistochemistry demonstrated that the expression of NLRC4 in the RTECs of patients with DN was higher compared with that of normal controls. In addition, the expression levels of PINK1, parkin and p-parkin, which are associated with mitophagy, were lower in the renal tissues of patients with DN compared with those in normal controls (Fig. 1). The increased expression of NLRC4 in the renal tubular epithelium of DN suggests that it may be involved in the injury of RTECs in DN. Previous studies have reported that mitophagy is dysregulated in DN RTECs $(17,26,27)$, and the present study also confirmed this finding. Furthermore, the expression of NLRC4 was found to be inversely proportional to the expression of mitochondrial autophagy-related proteins, suggesting that these may interact with each other.

$H G$-induced NLRC4 expression and secretion of the cytokines $I L-1 \beta$ and $I L-18$ are increased in HK2 cells. In order to investigate the effect of HG on the mitophagy of RTECs, PINK1, parkin and p-parkin expression levels were analyzed. MitoSOX was used to detect intracellular mROS. The results demonstrated that the expression levels of PINK1, parkin and p-parkin in HK2 cells treated with $30 \mathrm{mM}$ glucose (HG) for $48 \mathrm{~h}$ were lower compared with those in the $\mathrm{NG}$ and $\mathrm{HO}$ groups $(\mathrm{P}<0.05)$. In addition, the production of mROS was increased in the HG group. Thus, it was suggested that HG stimulation can cause mitophagy disorder and increase the production of mROS in RTECs. The results also indicated that HG stimulated the expression of NLRC4, secretion of IL-1 $\beta$ and IL-18 and HK2 cell death $(\mathrm{P}<0.05$; Fig. 2).

Knockdown of NLRC4 expression in HK2 cells treated with $H G$ can decrease the secretion of the inflammatory cytokines $I L-1 \beta$ and $I L-18$. Transfecting NLRC4 siRNA into HK 2 cells downregulated the expression of NLRC4. First, 
Table I. Clinical characteristics of patients with type 2 DN and control subjects.

\begin{tabular}{lcc}
\hline Characteristics & Control group $(\mathrm{n}=10)$ & Patients with DN $(\mathrm{n}=42)$ \\
\hline Sex, male/female $(\mathrm{n})$ & $6 / 4$ & $25 / 17$ \\
Age (years) & $53.0 \pm 6.3$ & $47.6 \pm 5.7$ \\
Duration of diabetes (years) & - & $8.1 \pm 7.4$ \\
Glycosylated hemoglobin $(\%)$ & $5.2 \pm 0.7$ & $6.8 \pm 2.2^{\mathrm{a}}$ \\
Hemoglobin $(\mathrm{g} / \mathrm{l})$ & $143.33 \pm 20.13$ & $116 \pm 26.2^{\mathrm{a}}$ \\
Serum creatinine $(\mu$ mol/l) & $82.67 \pm 16.26$ & $142.1 \pm 112.85^{\mathrm{a}}$ \\
Serum albumin $(\mathrm{g} / \mathrm{l})$ & $41.2 \pm 2.08$ & $35.80 \pm 9.02^{\mathrm{a}}$ \\
24-h urinary protein $(\mathrm{g} / 24 \mathrm{~h})$ & $0.10 \pm 0.04$ & $3.95 \pm 3.11^{\mathrm{a}}$ \\
Estimated glomerular filtration & $91.67 \pm 19.22$ & $67.84 \pm 36.96^{\mathrm{a}}$ \\
rate $\left(\mathrm{ml} / \mathrm{min} / 1.73 \mathrm{~m}^{2}\right)$ & &
\end{tabular}

Data are presented as mean $\pm \mathrm{SD}$. ${ }^{\mathrm{a}} \mathrm{P}<0.05$ vs. controls. $\mathrm{DN}$, diabetic nephropathy.
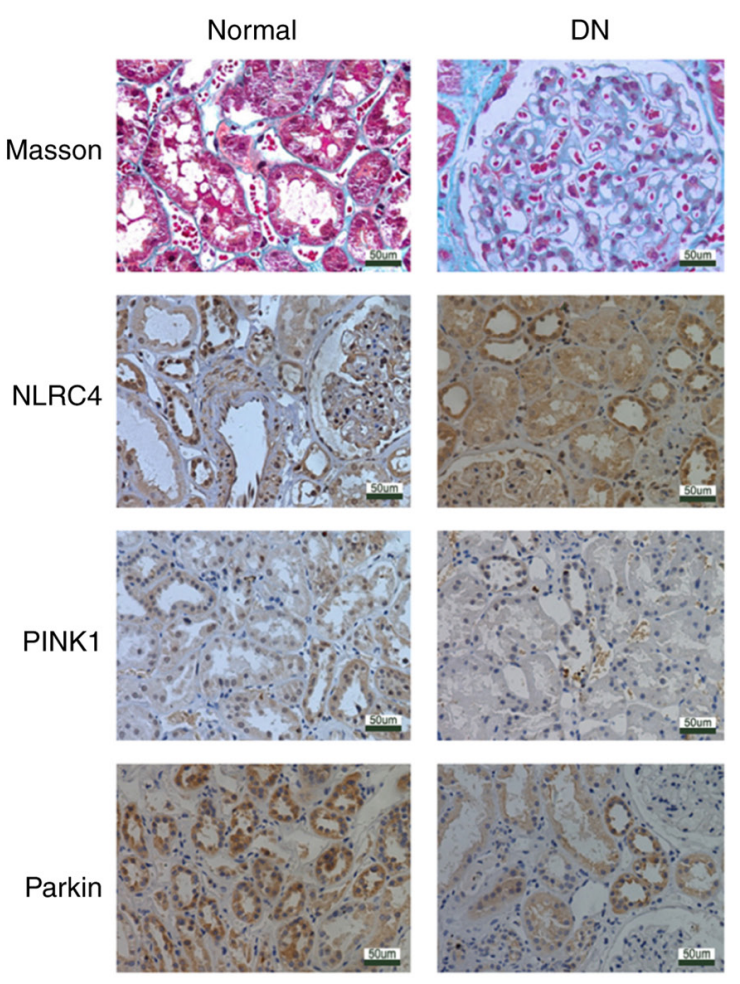
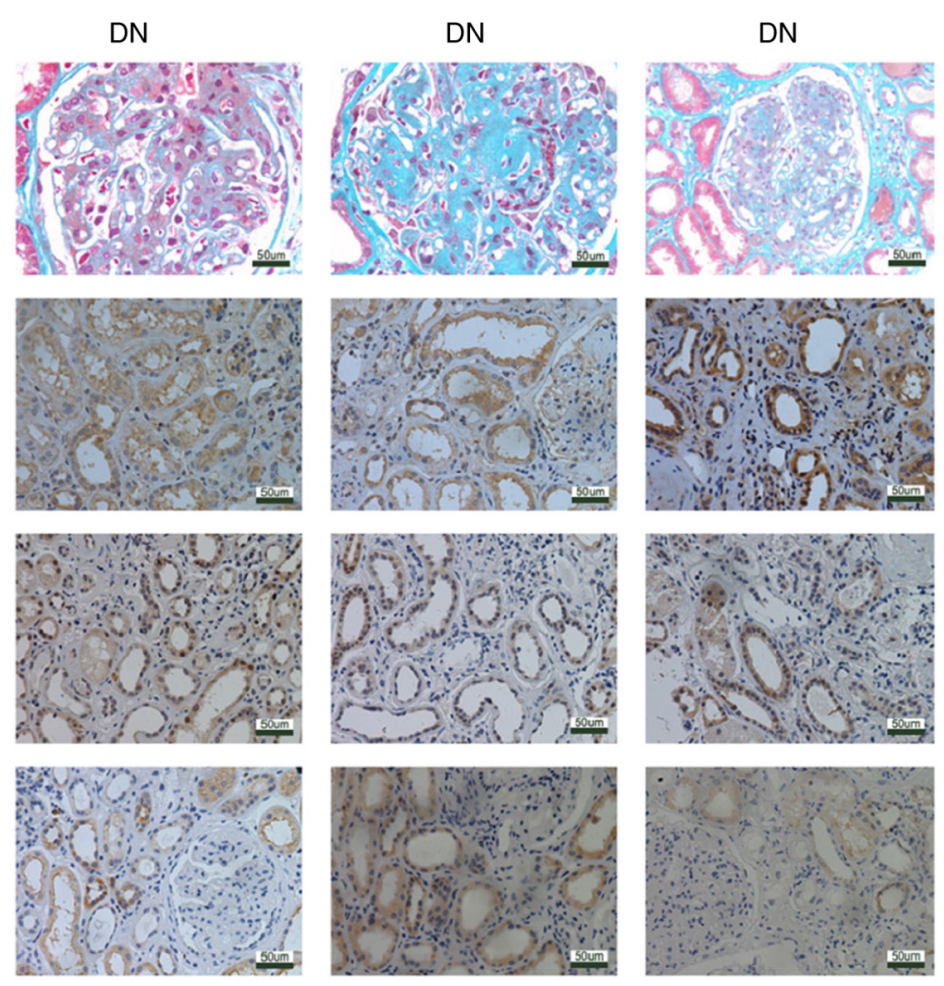

Figure 1. Immunohistochemical detection of NLRC4, PINK1 and parkin expression levels in renal tubule epithelial cells in control samples, and tissues from patients with type 2 DN (scale bars, $50 \mu \mathrm{m}$ ). NLRC4, NLR family CARD domain containing 4; PINK1, PTEN-induced kinase 1; DN, diabetic nephropathy.

the success of the transfection experiments of siNLRC4 was verified via western blotting in an NG environment (Fig. 3A). Then, these cells were divided into three groups: $\mathrm{HG}+$ siNLRC4, HG and control group. The cells were cultured for $48 \mathrm{~h}$. The results demonstrated that silencing NLRC4 expression in a $\mathrm{HG}$ environment could reduce the secretion of IL-1 $\beta$ and IL-18 and decrease HK2 cell death (Fig. 3). Thus, it was suggested that NLRC4 may be involved in HK2 cell death and the secretion of inflammatory cytokines in a HG environment. A schematic representation of the possible molecular mechanism linking disrupted mitophagy with NLRC4 inflammasome activation in RTECs under HG conditions is shown in Fig. 4.

\section{Discussion}

Several hypotheses have been suggested to explain the development and progression of DN; however, previous studies on DN mainly focused on glomerular injury (6). Recent studies have reported that DN glomerulopathy is important, but the role of renal tubular injury cannot be ignored $(6,28,29)$. Renal tubular injury may be an early lesion of $\mathrm{DN}$, and may predict and participate in the progression of DN (6). It was previously reported that, when the urinary albumin excretion rate of diabetic patients is normal, renal tubular injury is likely, indicating that renal tubular lesions may play an important role in the occurrence of DN (7). Brezniceanu et al (7) also 

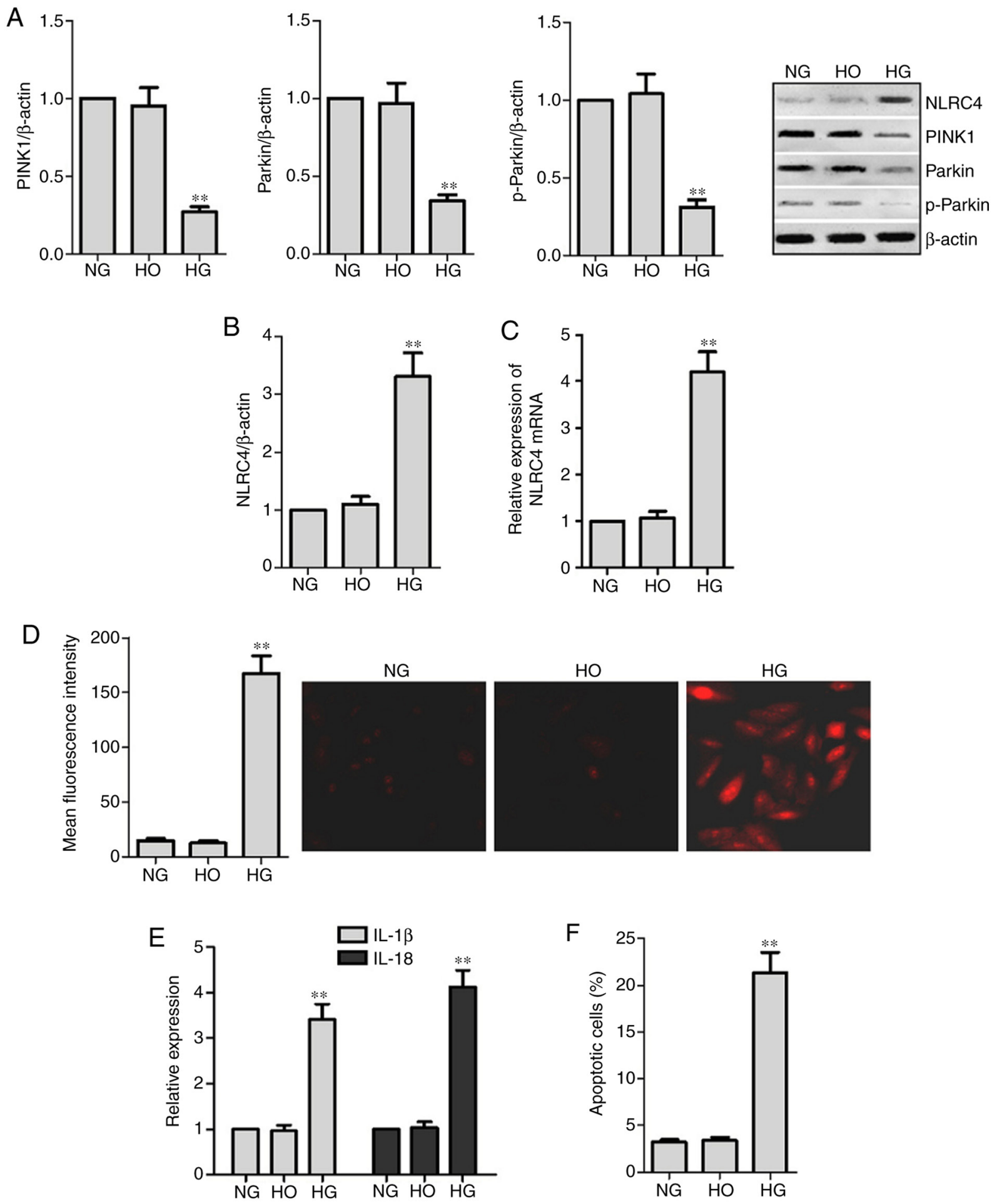

Figure 2. Effects of HG on mitophagy and NLRC4 inflammasome activation in HK2 cells. (A) Western blot analyses of PINK1, parkin and p-parkin levels in HK2 cells exposed to different glucose concentrations for 48 h. (B) NLRC4 protein expression was detected via western blot analysis; (C) NLRC4 mRNA expression was measured using reverse transcription-quantitative PCR in HK2 cells exposed to different glucose concentrations for 48 h. (D) Intracellular mROS were detected using MitoSOX. (E) ELISA of IL-1 $\beta$ and IL-18 levels in culture supernatants. (F) HK2 cell apoptosis under different glucose concentrations. ${ }^{* *} \mathrm{P}<0.01$ vs. control group. Both LSD and SNK post hoc tests were used after one-way ANOVA between different groups. NLRC4, NLR family CARD domain containing 4; PINK1, PTEN induced kinase 1; p-, phosphorylated; mROS, mitochondrial reactive oxygen species; HG, high glucose; NG, normal glucose (control); HO, hyperosmotic.

found that RTEC apoptosis and renal tubular atrophy may be present in the early stage of $\mathrm{DN}$, and the proximal renal tubule may be the earliest involved site. Therefore, the study of renal tubular injury in DN may uncover novel targets for the treatment of DN.
Most previous studies $(3,8,30)$ reported that $\mathrm{DN}$ is the result of the interaction of numerous factors, such as hemodynamic changes, metabolic disorders, oxidative stress and genetic factors, amongst others. In recent years, the role of inflammatory activation in the pathogenesis of DN has been 
A
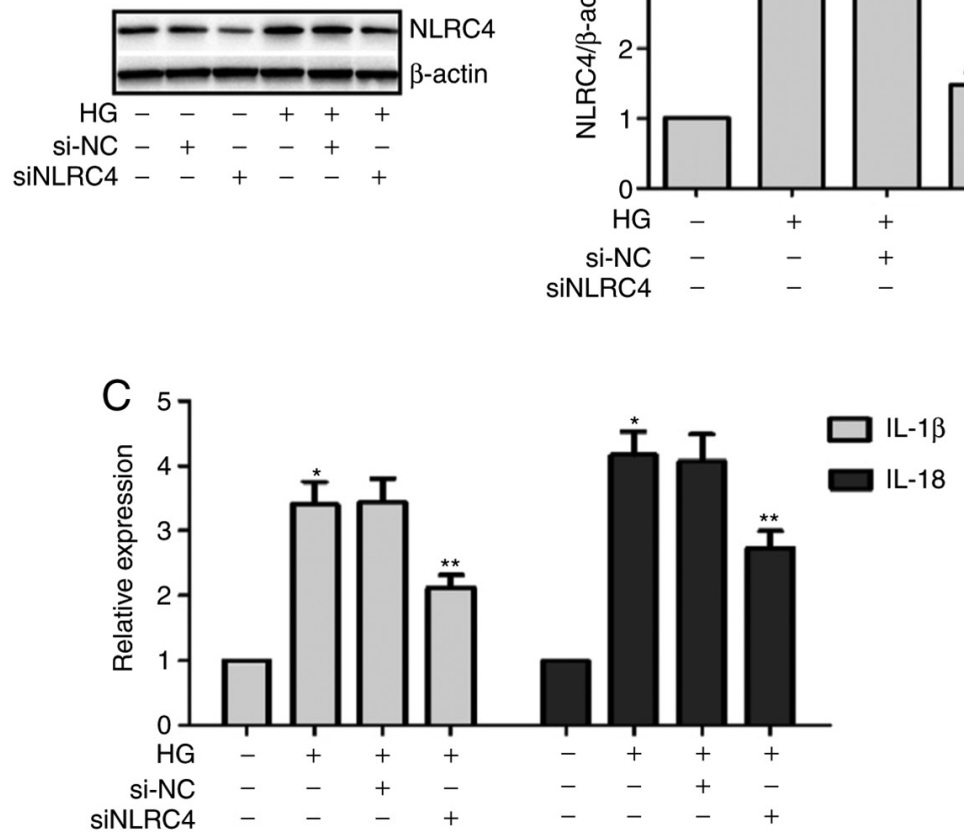
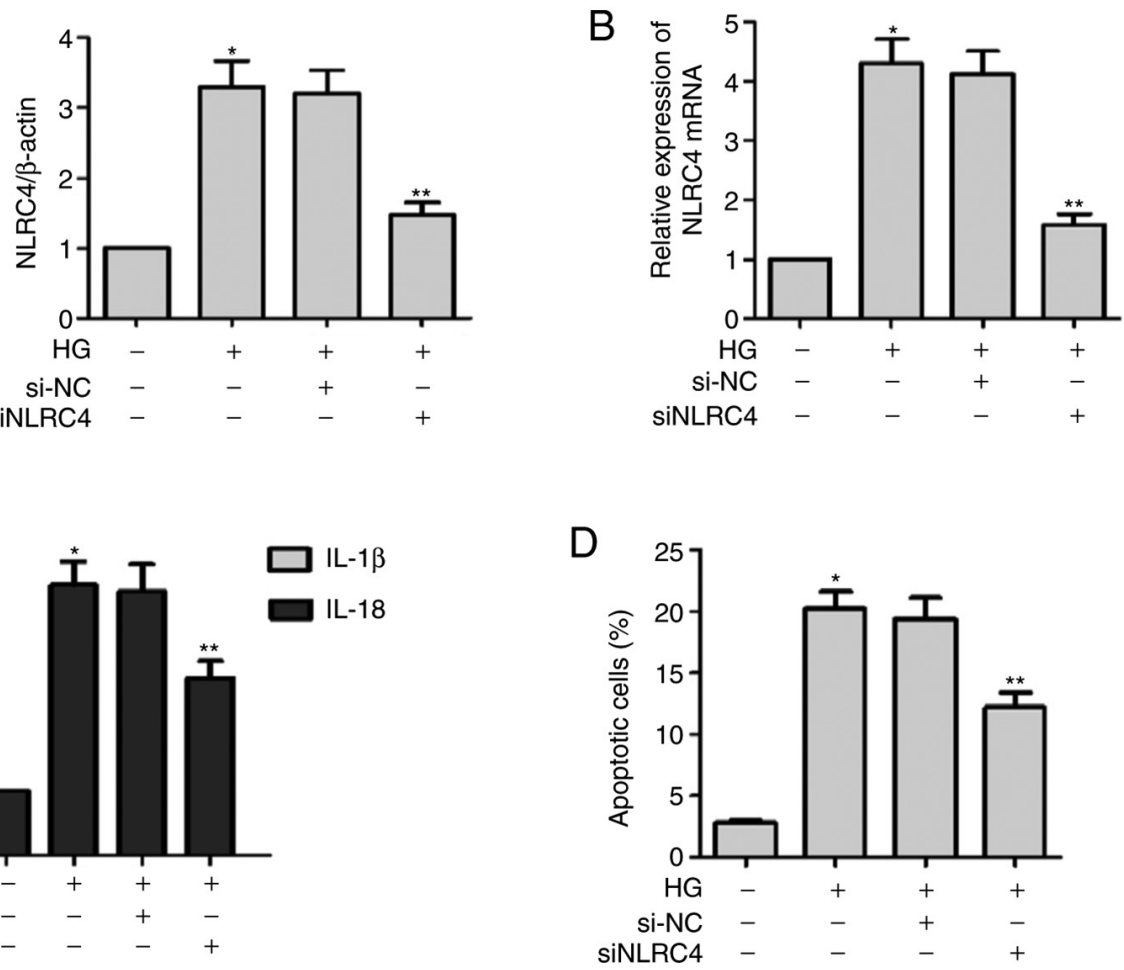

Figure 3. siRNA downregulates NLRC4 expression and NLRC4 inflammasome activation caused by HG. HK2 cells were incubated in 5.6 mM (control) or $30 \mathrm{mM}$ HG for $48 \mathrm{~h}$, with or without NLRC4 siRNA. (A) Western blot analysis and relative semi-quantitation of NLRC4 protein expression. (B) Reverse transcription-quantitative PCR analysis of NLRC4 mRNA expression. (C) ELISA of IL-1 $\beta$ and IL-18 levels in culture supernatants. (D) HK2 cell apoptosis under different glucose concentrations. ${ }^{*} \mathrm{P}<0.01$ vs. control group; ${ }^{* *} \mathrm{P}<0.05$ vs. HG group without NLRC4 siRNA. Both LSD and SNK post hoc tests were used after one-way ANOVA between different groups. NLRC4, NLR family CARD domain containing 4; siRNA, small interfering RNA; NC, negative control; HG, high glucose; NLRC4, NLR family CARD domain containing 4.

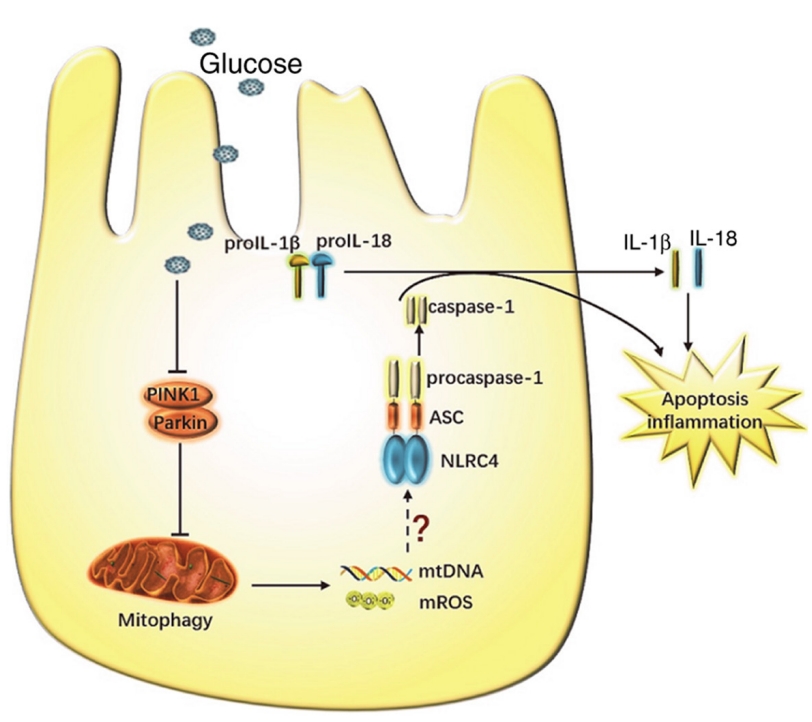

Figure 4. Possible molecular mechanism linking mitophagy disorder to NLRC4 inflammasome activation in renal tubular epithelial cells under high-glucose conditions. LRC4, NLR family CARD domain containing 4; mROS, mitochondrial reactive oxygen species; PINK1, PTEN induced kinase 1.

attracting increasing attention. The inflammatory response and activation of innate immunity serve an important role in the pathogenesis of DN (29). It has also been reported that the activation of the NLRP3 inflammasome is involved in the injury of RTECs in DN $(31,32)$, but the role of other inflammasomes in DN remains unknown. It has also been confirmed that bacterial products can activate the NLRC4 inflammasome (12). Moreover, some studies $(14,15,25)$ have observed that the NLRC4 inflammasome is involved in the occurrence of tumors, psoriasis and ulcerative colitis, indicating that the NLRC4 inflammasome plays an important role in the systemic inflammatory response and self-inflammatory diseases. The present study identified a causal link between NLRC4 inflammasome activation and RTEC injury.

The present study demonstrated that the expression of NLRC4 was increased in the RTECs of patients with DN and was associated with the grade of $\mathrm{DN}$, as the higher the grade, the higher the expression of NLRC4. In addition, the study of HK2 cells found that, under HG conditions, NLRC4 expression, IL-1 $\beta$ and IL-18 secretion and cell death were increased. Furthermore, it was found that knocking down the expression of NLRC4 in HK2 cells under HG conditions reduced the secretion of the inflammatory cytokines IL-1 $\beta$ and IL-18, and decreased cell death. Of note, flow cytometry may be more suitable for detecting apoptosis, and it will be perform in future experiments. It was also demonstrated that the NLRC4 inflammasome may be involved in the occurrence and development of DN, and its activation may not be solely caused by bacterial flagella or bacterial secreted substances. However, the factors that cause NLRC4 activation in DN remain to be fully determined.

Giacco and Brownlee (30) reported that in primary arterial endothelial cells in culture, intracellular hyperglycemia increases the voltage across the mitochondrial membrane to above the critical necessary threshold, which increases 
superoxide formation and, subsequently, enhances the production of ROS. It has been also revealed that dynamic changes in mitochondrial morphology are associated with HG-induced overproduction of ROS. Furthermore, Eleftheriadis et al (28) observed that HG increases solute carrier family 5 member 2 expression and glucose consumption, resulting in ROS overproduction. Thus, it was suggested that ROS may serve an important role in the pathogenesis of DN.

It was previously demonstrated that Pseudomonas aeruginosa infection can cause macrophage autophagy disturbance, accumulation of damaged mitochondria and increased production of mROS and mitochondrial DNA, which activates the NLRC4 inflammasome and increases the expression levels of caspase-1 and IL-1 $\beta$. Moreover, enhanced autophagy clearance of damaged mitochondria may significantly inhibit the activation of the NLRC4 inflammasome (20). Recently, it was reported that the disturbance of mitophagy in cardiomyocytes with heart failure after myocardial infarction in type 2 diabetic mice can promote the activation of the NLRC4 inflammasome, leading to an increase in IL-18 and cell death (21). The present study also demonstrated that mitophagy in RTECs was decreased in patients with DN, mitophagy in HK2 cells was impaired and NLRC4 expression was increased in a HG environment. These findings suggest that mitophagy may lead to the activation of the NLRC4 inflammasome and may be involved in cell injury via the production of mROS and mitochondrial RNA. However, additional research is required to validate these findings.

In conclusion, the present findings provided a rationale for developing targeted treatment methods for patients with DN by preventing inflammasome activation. It was suggested that mitophagy disorder may promote the activation of the NLRC4 inflammasome, but additional experiments are required to further confirm this result.

\section{Acknowledgements}

Not applicable.

\section{Funding}

The present study was supported by the National Natural Science Fund (grant no. U1904134).

\section{Availability of data and materials}

The datasets used and/or analyzed during the current study are available from the corresponding author on reasonable request.

\section{Authors' contributions}

YW, LT and RG designed, performed and analyzed the experiments. YW wrote and revised the manuscript. LY, LW, ZY and YG carried out the data collection, data analysis and revised the manuscript. LT revised the manuscript and applied for funding support. YW and LT confirm the authenticity of the raw data. All the authors have reviewed the results and approved the final version of the manuscript.

\section{Ethics approval and consent to participate}

The present study was approved by the Ethics Committee of The First Affiliated Hospital of Zhengzhou University (Zhengzhou, China). Written informed consent was obtained from each patient prior to participation in the study.

\section{Patient consent for publication}

Not applicable.

\section{Competing interests}

The authors declare that they have no competing interests.

\section{References}

1. Li Y, Teng D, Shi X, Qin G, Qin Y, Quan H, Shi B, Sun H, Ba J, Chen $\mathrm{B}$, et al: Prevalence of diabetes recorded in mainland China using 2018 diagnostic criteria from the American Diabetes Association: National cross sectional study. BMJ 369: m997, 2020.

2. Saeedi P,Petersohn I, Salpea P, Malanda B, Karuranga S, Unwin N, Colagiuri S, Guariguata L, Motala AA, Ogurtsova K, et al: Global and regional diabetes prevalence estimates for 2019 and projections for 2030 and 2045: Results from the International Diabetes Federation Diabetes Atlas, 9th edition. Diabetes Res Clin Pract 157: 107843, 2019.

3. Yang D, Livingston MJ, Liu Z, Dong G, Zhang M, Chen JK and Dong Z: Autophagy in diabetic kidney disease: Regulation, pathological role and therapeutic potential. Cell Mol Life Sci 75: 669-688, 2018.

4. Zhang L, Zhao MH, Zuo L, Wang Y, Yu F, Zhang H and Wang H; CK-NET Work Group: China kidney disease network (CK-NET) 2015 annual data report. Kidney Int Suppl (2011) 9: e1-e81, 2019.

5. Liu M, Liu SW, Wang LJ, Bai YM, Zeng XY, Guo HB, Liu YN, Jiang YY, Dong WL, He GX, et al: Burden of diabetes, hyperglycaemia in China from to 2016: Findings from the 1990 to 2016, global burden of disease study. Diabetes Metab 45: 286-293, 2019.

6. Gilbert RE: Proximal Tubulopathy: Prime mover and key therapeutic target in diabetic kidney disease. Diabetes 66: 791-800, 2017.

7. Brezniceanu ML, Liu F, Wei CC, Chénier I, Godin N, Zhang SL, Filep JG, Ingelfinger JR and Chan JS: Attenuation of interstitial fibrosis and tubular apoptosis in $\mathrm{db} / \mathrm{db}$ transgenic mice overexpressing catalase in renal proximal tubular cells. Diabetes 57: 451-459, 2008.

8. Tang SCW and Yiu WH: Innate immunity in diabetic kidney disease. Nat Rev Nephrol 16: 206-222, 2020.

9. Wu M, Han W, Song S, Du Y, Liu C, Chen N, Wu H, Shi Y and Duan H: NLRP3 deficiency ameliorates renal inflammation and fibrosis in diabetic mice. Mol Cell Endocrinol 478: 115-125, 2018.

10. Shahzad K, Bock F, Al-Dabet MM, Gadi I, Kohli S, Nazir S, Ghosh S, Ranjan S, Wang H, Madhusudhan T, et al: Caspase-1, but not caspase-3, promotes diabetic nephropathy. J Am Soc Nephrol 27: 2270-2275, 2016.

11. Song F, Ma Y, Bai XY and Chen X: The expression changes of inflammasomes in the aging rat kidneys. J Gerontol A Biol Sci Med Sci 71: 747-756, 2016.

12. Duncan JA and Canna SW: The NLRC4 inflammasome. Immunol Rev 281: 115-123, 2018.

13. Yuan F, Kolb R, Pandey G, Li W, Sun L, Liu F, Sutterwala FS, Liu Y and Zhang W: Involvement of the NLRC4-Inflammasome in diabetic nephropathy. PLoS One 11: e164135, 2016.

14. Rauch I, Deets KA, Ji DX, von Moltke J, Tenthorey JL, Lee AY, Philip NH, Ayres JS, Brodsky IE, Gronert K and Vance RE: NAIP-NLRC4 inflammasomes coordinate intestinal epithelial cell expulsion with eicosanoid and IL-18 release via activation of caspase-1 and -8. Immunity 46: 649-659, 2017.

15. Hiruma J, Harada K, Motoyama A, Okubo Y, Maeda T, Yamamoto M, Miyai M, Hibino T and Tsuboi R: Key component of inflammasome, NLRC4, was identified in the lesional epidermis of psoriatic patients. J Dermatol 45: 971-977, 2018. 
16. Lemasters JJ: Selective mitochondrial autophagy, or mitophagy, as a targeted defense against oxidative stress, mitochondrial dysfunction, and aging. Rejuvenation Res 8: 3-5, 2005.

17. Xiao L, Xu X, Zhang F, Wang M, Xu Y, Tang D, Wang J, Qin Y, Liu Y, Tang C, et al: The mitochondria-targeted antioxidant MitoQ ameliorated tubular injury mediated by mitophagy in diabetic kidney disease via Nrf2/PINK1. Redox Biol 11: 297-311, 2017.

18. Hamacher-Brady A and Brady NR: Mitophagy programs: Mechanisms and physiological implications of mitochondrial targeting by autophagy. Cell Mol Life Sci 73: 775-795, 2016.

19. Chen K, Dai H, Yuan J, Chen J, Lin L, Zhang W, Wang L, Zhang J, $\mathrm{Li} \mathrm{K}$ and $\mathrm{He} \mathrm{Y}$ : Optineurin-mediated mitophagy protects renal tubular epithelial cells against accelerated senescence in diabetic nephropathy. Cell Death Dis 9: 105, 2018.

20. Jabir MS, Hopkins L, Ritchie ND, Ullah I, Bayes HK, Li D, Tourlomousis P, Lupton A, Puleston D, Simon AK, et al: Mitochondrial damage contributes to Pseudomonas aeruginosa activation of the inflammasome and is downregulated by autophagy. Autophagy 11: 166-182, 2015.

21. Durga Devi T, Babu M, Mäkinen P, Kaikkonen MU, Heinaniemi M, Laakso H, Ylä-Herttuala E, Rieppo L, Liimatainen T, Naumenko N, et al: Aggravated postinfarct heart failure in type 2 diabetes is associated with impaired mitophagy and exaggerated inflammasome activation. Am J Pathol 187: 2659-2673, 2017.

22. Tervaert TW, Mooyaart AL, Amann K, Cohen AH, Cook HT, Drachenberg CB, Ferrario F, Fogo AB, Haas M, de Heer E, et al: Pathologic classification of diabetic nephropathy. J Am Soc Nephrol 21: 556-563, 2010.

23. Delanaye P and Pottel H: New equation to estimate glomerular filtration rate in China: A reference issue. Kidney Int 96: 521, 2019.

24. Livak KJ and Schmittgen TD: Analysis of relative gene expression data using real-time quantitative PCR and the 2(-Delta Delta C(T)) method. Methods 25: 402-408, 2001.
25. Janowski AM, Colegio OR, Hornick EE, McNiff JM, Martin MD, Badovinac VP, Norian LA, Zhang W, Cassel SL and Sutterwala FS: NLRC4 suppresses melanoma tumor progression independently of inflammasome activation. J Clin Invest 126 : 3917-3928, 2016.

26. Zhan M, Usman IM, Sun L and Kanwar YS: Disruption of renal tubular mitochondrial quality control by Myo-inositol oxygenase in diabetic kidney disease. J Am Soc Nephrol 26: 1304-1321, 2015.

27. Huang C, Zhang Y, Kelly DJ, Tan CY, Gill A, Cheng D, Braet F, Park JS, Sue CM, Pollock CA and Chen XM: Thioredoxin interacting protein (TXNIP) regulates tubular autophagy and mitophagy in diabetic nephropathy through the mTOR signaling pathway. Sci Rep 6: 29196, 2016.

28. Eleftheriadis T, Pissas G, Tsogka K, Nikolaou E, Liakopoulos V and Stefanidis I: A unifying model of glucotoxicity in human renal proximal tubular epithelial cells and the effect of the SGLT2 inhibitor dapagliflozin. Int Urol Nephrol 52: 1179-1189, 2020.

29. Gou R, Chen J, Sheng S, Wang R, Fang Y, Yang Z, Wang L and Tang L: KIM-1 mediates high glucose-induced autophagy and apoptosis in renal tubular epithelial cells. Cell Physiol Biochem 38: 2479-2488, 2016.

30. Giacco F and Brownlee M: Oxidative stress and diabetic complications. Circ Res 107: 1058-1070, 2010.

31. Chen K, Feng L, Hu W, Chen J, Wang X, Wang L and He Y: Optineurin inhibits NLRP3 inflammasome activation by enhancing mitophagy of renal tubular cells in diabetic nephropathy. FASEB J 33: 4571-4585, 2019.

32. Song S, Qiu D, Luo F, Wei J, Wu M, Wu H, Du C, Du Y, Ren Y, Chen N, et al: Knockdown of NLRP3 alleviates high glucose or TGFB1-induced EMT in human renal tubular cells. J Mol Endocrinol 61: 101-113, 2018. 